\title{
Paramètres structuraux et/ou ultrastructuraux facteurs de la variabilité intra-arbre de l'anisotropie élastique du bois
}

\author{
Daniel GuITARD, Christophe GACHET* \\ LEPT-ENSAM-UMR CNRS 8508-Université Bordeaux 1, Esplanade des Arts et Métiers, 33405 Talence Cedex, France
}

(Reçu le 18 janvier 2002 ; accepté le 16 décembre 2002)

\begin{abstract}
Résumé - La simulation de l'anisotropie élastique d'un bois résineux sans défaut est réalisée par empilement de trois échelles de descriptions, faisant passer successivement du niveau de la paroi cellulaire, à celui de chacun des tissus ligneux et enfin au niveau du cerne. Les modèles micromécaniques utilisés à chaque étape restent basés sur la recherche du solide élastique homogène équivalent, par des lois des mélanges simples appliquées à des assemblages série ou parallèle de sous domaines supposés homogènes. Un jeu utile de 17 paramètres réalistes, en regard de données bibliographiques variées, est optimisé sur la base des données caractéristiques élastiques anisotropes du «résineux standard ». L'outil de modélisation est adapté à l'analyse de la sensibilité des propriétés élastiques à la variabilité des paramètres adoptés bien que s'éloignant quelque peu de la stricte réalité ultrastructurale. Une originalité réside dans la schématisation de la paroi cellulaire en une couche unique, renforcée par un squelette de microfibrilles, dont l'orientation est empruntée à l'angle des microfibrilles de la sous couche $\mathrm{S}_{2}$; le caractère rigidifiant du squelette, par comparaison à une structure renforcée par des fibres parallèles traduit les renforcements transverses de la paroi, le plus souvent imputés aux sous couches $S_{1}$ et $S_{3}$.
\end{abstract}

bois / anisotropie / élasticité / micromécanique / ultrastructure

Abstract - Structural and/or ultrastructural parameters governing the variability inside the tree of the elastic anisotropy of wood. Three levels of modelling are introduced to simulate the elastic anisotropy of solid clear softwood, from the cell wall level, via wooden tissues, to the annual ring level. The micro-mechanical models used, at each step, are based on the homogeneous elastic equivalent solid method. Quite simple mixture laws are used for series or parallels assembling of two assumed homogeneous media. 17 realistic parameters are identified by optimisation on the base of given orthotropic elastic properties of the "standard softwood". The modelling tool allows analysing the sensibility of elastic parameters to the variability of internal structural parameters even if it doesn't express quite the ultrastructural reality. One of the originalities is include in cellular wall schematization by a unique layer, reinforcing by a microfibrille skeleton, in which the microfibrillar orientation belongs to $S_{2}$ layer. The stiffening of this skeleton, in front of a structure with parallels fibers, represents the cell walls tranverses reinforcements generally assigned to $S_{2}$ and $S_{3}$ layers.

wood / anisotropy / elasticity / micro mechanics / ultrastructure

\section{INTRODUCTION}

Depuis longtemps, de nombreux auteurs (tels Carrington en 1922, Kollmann et al. en 1941 et 1960 rapportés dans [22], plus récemment $[4,16,17])$ se sont préoccupés d'analyser et même de modéliser l'influence de paramètres physiques tels que la masse volumique, le taux d'humidité, ou encore la température sur les propriétés élastiques du matériau bois.

Des modèles prévisionnels donnant les neuf constantes de la matrice d'élasticité orthotrope, s'appuyant sur des indicateurs tels que la nature feuillue ou résineuse de l'essence considérée, la masse volumique, le taux d'humidité et la température, construits sur les bases d'une banque de données de propriétés matérielles d'origines très diverses [16], ont démontré leur pertinence quand à l'évaluation, à priori, des propriétés d'un bois «normal »sans défaut. En effet, plus de $80 \%$ de la variabilité intra spécifique, et même inter spécifique, de ces caractéristiques sont largement expliqués au moyen de ces seuls indicateurs.

Paradoxalement, ces modèles sont mis en défaut pour décrire, au sein d'un même arbre, la variabilité des propriétés des différents tissus ligneux (bois juvénile, bois initial, bois final, bois de réaction ou encore duramen).

Il s'agit ici, en s'appuyant sur une large étude bibliographique, de procéder à un inventaire des paramètres pertinents, aux différentes échelles (de l'ultrastructure pariétale à la macrostructure

* Auteur pour correspondance : gachet@lept-ensam.u-bordeaux.fr 
que constitue le plan ligneux), susceptibles de conditionner les propriétés élastiques anisotropes du bois sans défaut, d'intégrer ces paramètres dans une modélisation prévisionnelle de type micro-macro, laquelle, par des essais numériques, permettra de procéder à une hiérarchisation de leurs influences sur la variabilité des propriétés élastiques.

\section{INVENTAIRES DE PARAMÈTRES FACTEURS DE L'ANISOTROPIE ÉLASTIQUE}

Les différentes échelles de description de la matière ligneuse sont ici successivement explorées, depuis l'ultrastructure, jusqu'à la macrostucture du bois massif sans défaut, dans une perspective mécanicienne. Les éléments bibliographiques essentiels sont évoqués en appui des choix retenus in fine, dans l'objectif d'alimenter un empilement de modèles micro-macro explicatifs et descriptifs du comportement élastique anisotrope macroscopique du bois massif. S'agissant de considérer un grand nombre de paramètres, pertinents à différentes échelles, ceux-ci sont intégrés dans des modèles élémentaires non nécessairement les plus sophistiqués les prenant en compte, pour le moins au premier ordre.

\subsection{La proportion de cellulose cristalline}

La matière constitutive de la paroi cellulaire est considérée comme un mélange de macromolécules de plusieurs biopolymères ; pour l'essentiel de la lignine, des hémicelluloses et de la cellulose [1, 4, 7, 14, 27, 28].

Si la lignine et les hémicelluloses constituent les composants d'une matrice amorphe, une fraction volumique importante de la cellulose cristalline est présente sous forme de microfibrilles orientées. En accord avec de nombreux auteurs, la matière constitutive de la paroi cellulaire est assimilée à un matériau composite à deux composantes : un renfort filamentaire, constitué par un squelette de microfibrilles de cellulose, noyé dans une matrice amorphe de lignine et d'hémicelluloses.

Les paramètres micromécaniques retenus à cette échelle sont les suivants :

- propriétés élastiques isotropes de la matrice :

$\mathrm{E}^{\mathrm{m}}$ : module d'Young,

$v$ : coefficient de Poisson ;

- propriétés élastiques des microfibrilles de cellulose :

$\mathrm{E}^{\mathrm{f}}$ : module d'élasticité longitudinal.

Notons qu'un comportement élastique anisotrope des microfibrilles a été envisagé [19], toutefois un tel niveau de complexité ne sera pas retenu ici.

Une grandeur caractéristique de la proportion de renfort filamentaire est adoptée :

$\mathrm{V}$ : fraction volumique en microfibrilles.

\subsection{La paroi cellulaire renforcée par des microfibrilles orientées}

Les descriptions cytologiques pariétales de la trachéide distinguent généralement les sous couches $S_{1}, S_{2}$ et $S_{3}$ constituant la paroi secondaire, la liaison intercellulaire étant assurée par la lamelle mitoyenne LM fortement lignifiée [27, 28].
Il est généralement observé que la sous couche $S_{2}$ est la plus épaisse et représente près de $80 \%$ de l'épaisseur pariétale totale. De plus, exception faite de la sous couche $\mathrm{S}_{3}$, dite couche gélatineuse, du bois de réaction des feuillus, $S_{2}$ est la plus riche en cellulose. C'est d'autre part dans $S_{2}$ que les microfibrilles quasi parallèles ont une orientation privilégiée. Le paramètre Angle des Microfibrilles (AMF) caractérise l'inclinaison des microfibrilles dans la paroi par rapport au grand axe de la cellule. Pour ces raisons, la modélisation de la paroi cellulaire utilisée dans la suite fait jouer un rôle prépondérant à la sous couche $S_{2}$.

Le paramètre essentiel retenu ici est l'angle des microfibrilles :

$\varphi:(\mathrm{AMF})$ angle des microfibrilles.

Différents modèles ont été proposés pour décrire l'anisotropie élastique pariétale à partir des propriétés élastiques des constituants de la paroi et de l'AMF $\varphi$. Ils considèrent généralement la juxtaposition de deux parois, appartenant respectivement à deux cellules adjacentes [1, 4, 7, 8, 14], Norimoto 1986 dans [15, 23, 24], prenant éventuellement en compte les sous couches $S_{1}$ et une lamelle mitoyenne $M$. Les couches externes du multicouche, représentatives des sous couches $S_{2}$, sont à fibres inclinées à $\pm \varphi$ par rapport à l'axe cellulaire (Fig. 1 ).

Dans un esprit voisin, un modèle micromécanique, proposé par Guitard [19], a été élaboré en assimilant le comportement élastique d'un tissus ligneux composé d'un ensemble de plusieurs cellules jointives (et non pas d'une cellule isolée), à celui d'une cellule virtuelle unique, possédant un squelette tridimensionnel de microfibrilles, constitué d'un double enroulement de microfibrilles d'orientation $\pm \varphi$, noyé dans une matrice élastique amorphe.

Il fournit notamment des estimations des rigidités suivant les directions longitudinale $\mathrm{E}_{\mathrm{LP}}$ (1) et transverses $\mathrm{E}_{\mathrm{TP}}$ et $\mathrm{E}_{\mathrm{RP}}$ (2) de la paroi cellulaire [19], ceci en fonction des paramètres caractéristiques des propriétés pariétales indiqués ci-dessus (\$ 2.1).

$$
\begin{aligned}
\mathrm{E}_{\mathrm{LP}} \approx \mathrm{C}_{\mathrm{zzzz}}= & (1-\mathrm{V}) \frac{(1-v)}{(1+v)(1-2 v)} \mathrm{E}^{\mathrm{m}} \\
+ & \mathrm{V} \cos ^{2}(\varphi)\left(1+\sin ^{2}(\varphi)\right) \mathrm{E}^{\mathrm{f}} \\
\mathrm{E}_{\mathrm{TP}}=\mathrm{E}_{\mathrm{RP}} \approx \mathrm{C}_{\theta \theta \theta \theta} & =(1-\mathrm{V}) \frac{(1-v)}{(1+v)(1-2 v)} \mathrm{E}^{\mathrm{m}} \\
& +\mathrm{V} \sin ^{2}(\varphi)\left(1+\cos ^{2}(\varphi)\right) \mathrm{E}^{\mathrm{f}} .
\end{aligned}
$$

Certains modèles micro-macro plus complexes, prenant en compte avec un plus grand nombre de paramètres le caractère multicouche de la paroi cellulaire et notamment la sous-couche $\mathrm{S}_{1}$, ont été proposés par ailleurs [15]. Ce niveau de sophistication n'a pas été retenu au stade de la présente étude.

Le modèle présenté ici a en effet montré sa pertinence quand à la prédiction des déformations résiduelles de maturation tant longitudinales (DRLM) que tangentielles (DRTM) à partir des mesures d'AMF (inversion de la DRLM), sans que l'intervention des autres sous couches ne soit rendue nécessaire.

\subsection{Au niveau du tissu ligneux : la morphologie cellulaire}

Le niveau d'observation considéré dans ce paragraphe est celui d'un assemblage de trachéides identiques constituant un tissu ligneux homogène. L'observation d'une micrographie de 


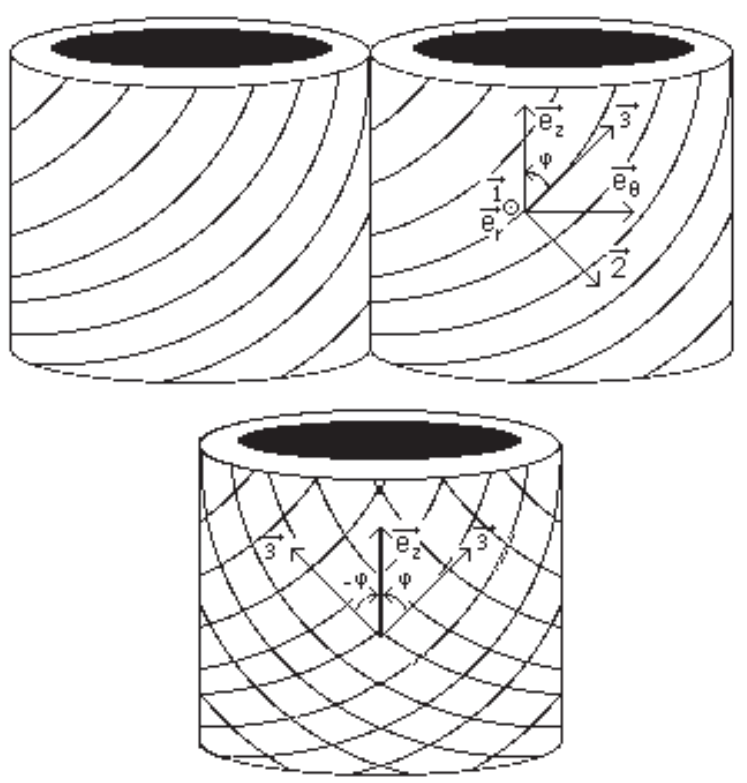

Figure 1. Une cellule unique avec un double enroulement croisé de nappes de microfibrilles représentative du tissu ligneux.
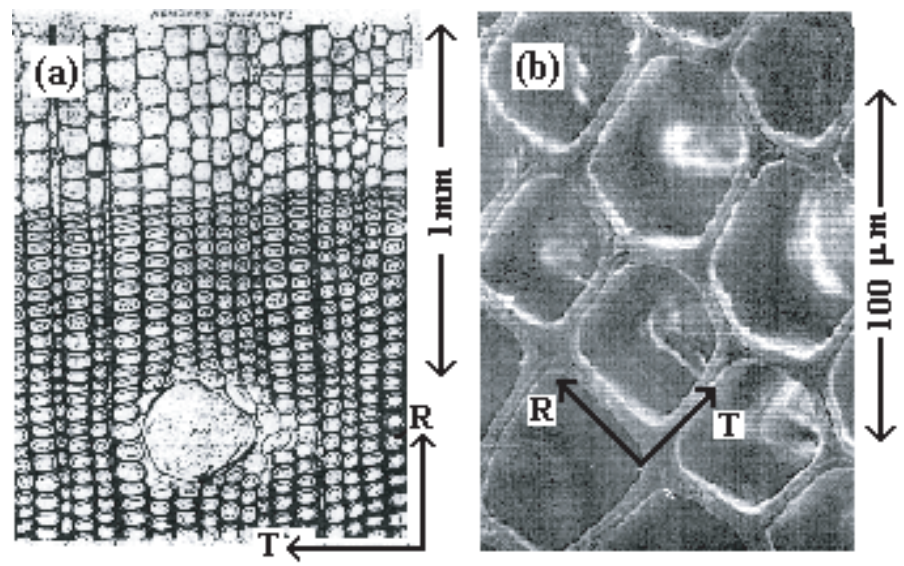

Figure 2. Micrographies d'une section radiale-tangentielle de résineux. (a) Limite de cerne entre bois final et bois initial. (b) Alignement suivant $R$, quinconces suivant $T$.

section radiale-tangentielle de résineux (Figs. 2a, b) suggère très naturellement $d$ 'assimiler l'assemblage cellulaire à une structure de type nid d'abeille. Le modèle proposé par Gibson [13] est une cellule à section droite hexagonale caractérisée par quatre paramètres, indiqués sur la figure $3 \mathrm{a}$. Nous adoptons ici un modèle rectangulaire apparemment plus simple (Fig. 3b). Toutefois, ce modèle, comme le précédent est à quatre paramètres :

$\mathrm{D}_{\mathrm{R}}$ : diamètre cellulaire radial,

$\mathrm{e}_{\mathrm{R}}$ : épaisseur pariétale radiale,

$\mathrm{D}_{\mathrm{T}}$ : diamètre cellulaire tangentiel,

$\mathrm{e}_{\mathrm{T}}$ : épaisseur pariétale tangentielle.
Notons que le paramètre $\theta$ du modèle de Gibson, donnant la direction de la paroi cellulaire radiale par rapport à la direction radiale du bois (Fig. 3a), est ici pris égal à zéro. L'influence de ce paramètre est prise en compte de façon indirecte en considérant que l'épaisseur $\mathrm{e}_{\mathrm{R}}$ de la paroi radiale peut être différente de l'épaisseur $\mathrm{e}_{\mathrm{T}}$ de la paroi tangentielle. Dans ces conditions, il est possible d'évaluer les modules élastiques du solide homogène équivalent selon les trois directions du nid d'abeille.

\subsubsection{Le module d'élasticité longitudinal $E_{L}^{*}$}

Le module d'élasticité longitudinal $\mathrm{E}_{\mathrm{L}}^{*}$ homogénéisé du tissus ligneux s'évalue à partir du module pariétal longitudinal 


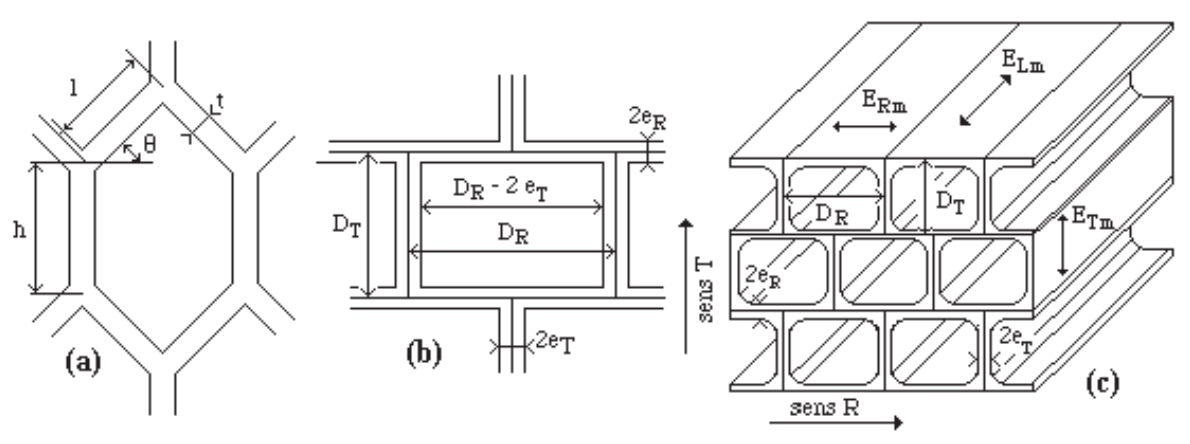

Figure 3. (a) Modèle de Gibson, (b) notre modèle.

$\mathrm{E}_{\mathrm{LP}}$, en proportion de l'aire de surface active de la paroi, par rapport à l'aire apparente de la section droite de cellule (Fig. 3b), soit :

$$
\begin{aligned}
E_{L}^{*} & =\frac{\left[D_{R} D_{T}-\left(D_{R}-2 e_{T}\right)\left(D_{T}-2 e_{R}\right)\right]}{D_{R} D_{T}} E_{L P} \\
& =2\left[\frac{e_{T}}{D_{R}}+\frac{e_{R}}{D_{T}}+2 \frac{e_{T} e_{R}}{D_{R} D_{T}}\right] E_{L P} .
\end{aligned}
$$

Notons que le coefficient multiplicatif de $\mathrm{E}_{\mathrm{LP}}$ dans (3) est en relation directe avec la porosité $\mathrm{P}_{0}$ du nid d'abeille, comme le montre la relation (4), dans laquelle, $\rho$ désigne la masse volumique apparente, tandis que $\rho_{\mathrm{P}}$ représente la masse volumique de la paroi cellulaire considérée comme sensiblement constante $\left(\rho_{\mathrm{P}} \approx 1,51 \mathrm{~g} \cdot \mathrm{cm}^{-3}\right)$.

$$
\rho=\left(1-\mathrm{P}_{0}\right) \rho_{\mathrm{P}}=2\left[\frac{\mathrm{e}_{\mathrm{T}}}{\mathrm{D}_{\mathrm{R}}}+\frac{\mathrm{e}_{\mathrm{R}}}{\mathrm{D}_{\mathrm{T}}}-2 \frac{\mathrm{e}_{\mathrm{T}} \mathrm{e}_{\mathrm{R}}}{\mathrm{D}_{\mathrm{R}} \mathrm{D}_{\mathrm{T}}}\right] \rho_{\mathrm{P}} .
$$

Dans ces conditions (3) et (4) font que $\mathrm{E}_{\mathrm{L}}^{*}$ est proportionnel à la masse volumique $\rho$.

$$
\mathrm{E}_{\mathrm{L}}^{*}=\frac{\rho}{\rho_{\mathrm{P}}} \mathrm{E}_{\mathrm{LP}} .
$$

En conséquence, on notera que, compte tenu de la donnée de $\rho_{P}$, la masse volumique apparente $\rho$ est un paramètre lié aux 4 caractéristiques géométriques du nid d'abeille retenues ci dessus.

\subsubsection{Le module d'élasticité tangentiel $E_{T}^{*}$}

Le module d'élasticité $\mathrm{E}_{\mathrm{T}}^{*}$, selon le sens $\mathrm{T}$ du nid d'abeille, est évalué en considérant la maille élémentaire dans le repère $(\mathrm{C}, \overrightarrow{\mathrm{x}}, \overrightarrow{\mathrm{y}})$ représentée sur la figure 4 . La configuration choisie correspond à un arrangement en quinconce des cellules.

La souplesse exprimée en (6) résulte de la combinaison de la souplesse des éléments AB, FE et CD de la maille sollicités en traction-compression et de celle de l'élément de paroi BCE travaillant, quant à lui, en flexion trois points.

$$
\frac{1}{\mathrm{E}_{\mathrm{T}}^{*}}=\left[1+\frac{1}{16} \frac{\mathrm{E}_{\mathrm{TP}}}{\mathrm{E}_{\mathrm{RP}}} \frac{\mathrm{e}_{\mathrm{T}}}{\mathrm{D}_{\mathrm{T}}} \frac{\left(\mathrm{D}_{\mathrm{R}}-2 \mathrm{e}_{\mathrm{T}}\right)^{3}}{\mathrm{e}_{\mathrm{R}}^{3}}\right] \frac{\mathrm{D}_{\mathrm{R}}}{2 \mathrm{e}_{\mathrm{T}}} \frac{1}{\mathrm{E}_{\mathrm{TP}}} .
$$
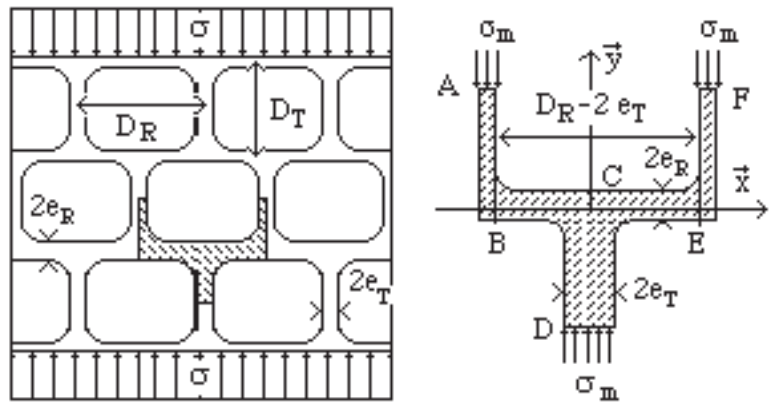

Figure 4. Évaluation du module $\mathrm{E}_{\mathrm{T}}$, sollicitation dans le sens $\mathrm{T}$ de la maille élémentaire.

On notera que la longueur de paroi fléchie $\left(\mathrm{D}_{\mathrm{R}}-2 \mathrm{e}_{\mathrm{T}}\right)$, prise en compte dans le modèle (6) est égale au diamètre $\mathrm{D}_{\mathrm{R}}$, diminué de deux fois l'épaisseur de paroi $\mathrm{e}_{\mathrm{T}}$. Ceci constitue une autre différence par rapport au modèle proposé par Gibson [13] dans lequel la longueur de paroi fléchie est prise égale au diamètre cellulaire $\mathrm{D}_{\mathrm{R}}$, ceci indépendamment de l'épaisseur pariétale. L'élément que nous avons retenu est en conséquence plus rigide, notamment dans le cas de tissus ligneux de masse volumique élevée et donc à parois épaisses.

\subsubsection{Le module d'élasticité radial $E_{R}^{*}$}

Le module d'élasticité homogénéisé $\mathrm{E}_{\mathrm{R}}^{*}$, selon le sens $\mathrm{R}$ du nid d'abeille, prend en compte la rigidité en traction-compression des éléments de paroi radiale ECB, disposés dans le prolongement les uns des autres, comme illustré sur la figure 5. Le module d'élasticité $\mathrm{E}_{\mathrm{R}}^{*}(7)$ est proportionnel à $\mathrm{E}_{\mathrm{RP}}$, dans le rapport de l'épaisseur de paroi radiale $\mathrm{e}_{\mathrm{R}}$ au diamètre tangentiel $\mathrm{D}_{\mathrm{T}}$. Soit :

$$
\mathrm{E}_{\mathrm{R}}^{\mathrm{x}}=\frac{2 \mathrm{e}_{\mathrm{R}}}{\mathrm{D}_{\mathrm{T}}} \mathrm{E}_{\mathrm{RP}} .
$$

Notons qu'aucun élément de paroi n'est sollicité en flexion, dans le cas d'une sollicitation de sens R.

Dans la suite, et de façon quelque peu originale, les différents tissus ligneux constitutifs d'un bois donné seront caractérisés par des jeux spécifiques de paramètres pariétaux et de paramètres 

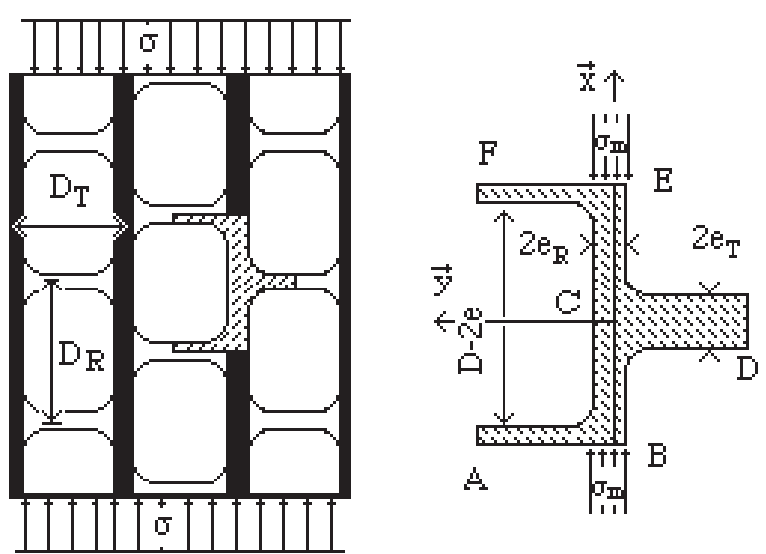

Figure 5. Évaluation du module $\mathrm{E}_{\mathrm{R}}$, sollicitation suivant le sens $\mathrm{R}$.

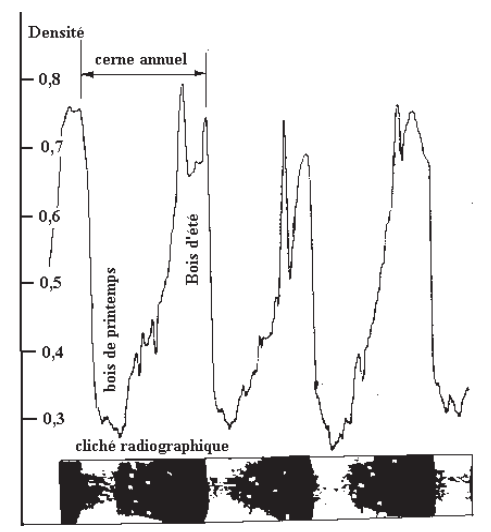

Figure 6. Profil microdensitométrique de quatre cernes selon la direction radiale.

géométriques tissulaires, permettant alors de différentier : bois initial, bois final, bois de réaction, etc.

\subsection{Hétérogénéité au niveau du cerne de croissance}

L'échelle d'observation immédiatement supérieure se situe au niveau du cerne de croissance. Le cliché radiographique figure 6 illustre par un profil microdensitométrique l'alternance dans le sens radial des couches de bois initial de faible masse volumique, voisine de $0,25 \mathrm{~g} \cdot \mathrm{cm}^{-3}$, et de bois final de masse volumique $\rho$ plus élevée, atteignant $0,75 \mathrm{~g} \cdot \mathrm{cm}^{-3}$. Pour décrire plus précisément le plan ligneux, il convient d'adjoindre aux deux tissus, bois initial et bois final, un troisième tissu constitué par les rayons ligneux, visibles sur le cliché figure 2a. La maille de base des trois tissus est schématisée sur la figure 7. La prise en compte de ces trois tissus ligneux pour modéliser le comportement élastique d'un bois propre est réalisée en deux étapes. Une première étape consiste à exprimer les propriétés homogénéisées du bicouche (bois «infi») que constitue l'alternance bois initial, bois final, en introduisant le paramètre de texture du cerne. Une seconde étape consiste à rechercher les propriétés homogénéisées du bicouche que constitue le bois

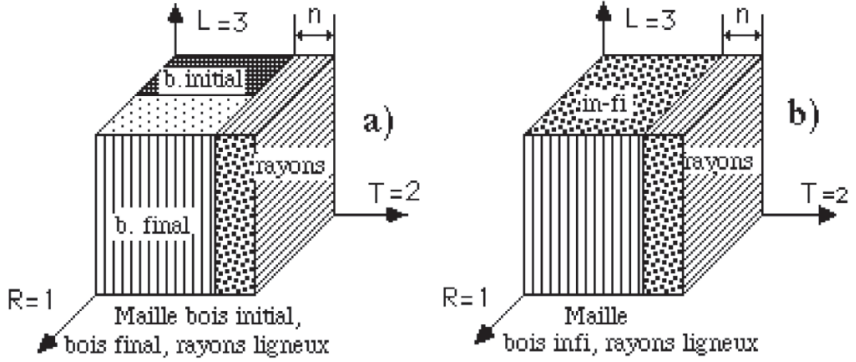

Figure 7. Mailles élémentaires, avec passage d'un modèle à trois tissus à un modèle à deux tissus.

« infi » (précédemment conçu) et le tissu rayons ligneux, en introduisant cette fois le paramètre donnant la proportion de rayons ligneux dans l'essence considérée.

\subsubsection{Prise en compte de la texture $T_{X}$}

Le cerne est assimilé à un bicouche de bois initial et de bois final (Fig. 7), caractérisé par la texture (Tx), rapport de la largeur de bois final à la largeur de cerne. Les propriétés élastiques homogénéisées du bois «infi » sont évaluées, par une simple loi des mélanges, à partir de celles des tissus constitutifs (bois initial et bois final). Une association série de rigidités est envisagée pour le sens radial $\mathrm{R}$ et une association en parallèle pour les directions tangentielle $\mathrm{T}$ et longitudinale $\mathrm{L}$.

$$
\begin{gathered}
\frac{1}{\mathrm{E}_{\mathrm{R}}^{\text {infi }}}=\frac{(1-\mathrm{Tx})}{\mathrm{E}_{\mathrm{R}}^{\text {initial }}}+\frac{\mathrm{Tx}}{\mathrm{E}_{\mathrm{R}}^{\text {final }}}, \\
\mathrm{E}_{\mathrm{T}}^{\text {infi }}=(1-\mathrm{Tx}) \cdot \mathrm{E}_{\mathrm{T}}^{\text {initial }}+\mathrm{Tx} \cdot \mathrm{E}_{\mathrm{T}}^{\text {final }}, \\
\mathrm{E}_{\mathrm{L}}^{\text {infi }}=(1-\mathrm{Tx}) \cdot \mathrm{E}_{\mathrm{L}}^{\text {initial }}+\mathrm{Tx} \cdot \mathrm{E}_{\mathrm{L}}^{\mathrm{final}},
\end{gathered}
$$

$\mathrm{E}_{\mathrm{R}}^{\text {infi }}, \mathrm{E}_{\mathrm{T}}^{\text {infi }}, \mathrm{E}_{\mathrm{L}}^{\text {infi }}$ : modules d'élasticité du bicouche (bois « infi »). $\mathrm{E}_{\mathrm{R}}^{\mathrm{X}}, \mathrm{E}_{\mathrm{T}}^{\mathrm{X}}, \mathrm{E}_{\mathrm{L}}^{\mathrm{X}}$ : modules d'élasticité de la couche $\mathrm{x}$ (initial, final). $\mathrm{Tx}=\frac{\mathrm{L}_{\text {final }}}{\mathrm{L}_{\text {cerne }}}$ : texture, rapport de l'épaisseur de bois final $\mathrm{L}_{\text {final }}$ à la largeur de cerne $\mathrm{L}_{\text {cerne }}$.

\subsubsection{Fraction en rayons ligneux facteur d'anisotropie transverse}

Les rayons ligneux, orientés en quadrature par rapport aux cellules de bois initial et final, représentent le troisième tissu constitutif du plan ligneux. La figure 7 schématise l'assemblage d'un tissu de rayons ligneux et du bicouche « infi » homogénéisé, précédemment défini.

Les propriétés élastiques homogénéisées du cerne, incluant le tissu rayon ligneux, sont évaluées, encore par une simple loi des mélanges, à partir de celles des tissus constitutifs (bois « infi » et rayons), moyennant le paramètre $n$ caractérisant la fraction volumique en rayons ligneux. Une association série de rigidités est envisagée pour le sens tangentiel T et une association en parallèle pour les directions longitudinale $\mathrm{L}$ et radiale $\mathrm{R}$. 


$$
\begin{gathered}
\mathrm{E}_{\mathrm{R}}=(1-\mathrm{n}) \cdot \mathrm{E}_{\mathrm{L}}^{\text {infi }}+\mathrm{n} \cdot \mathrm{E}_{\mathrm{R}}^{\text {rayon }}, \\
\frac{1}{\mathrm{E}_{\mathrm{T}}}=\frac{(1-\mathrm{n})}{\mathrm{E}_{\mathrm{T}}^{\text {infi }}}+\frac{\mathrm{n}}{\mathrm{E}_{\mathrm{T}}^{\text {rayon }}}, \\
\mathrm{E}_{\mathrm{L}}=(1-\mathrm{n}) \cdot \mathrm{E}_{\mathrm{L}}^{\text {infi }}+\mathrm{n} \cdot \mathrm{E}_{\mathrm{L}}^{\text {rayon }},
\end{gathered}
$$

$\mathrm{E}_{\mathrm{R}}, \mathrm{E}_{\mathrm{T}}, \mathrm{E}_{\mathrm{L}}$ : modules d'élasticité homogénéisés du bois propre. $\mathrm{E}_{\mathrm{R}}^{\text {infi }}, \mathrm{E}_{\mathrm{T}}^{\text {infi }}, \mathrm{E}_{\mathrm{L}}^{\text {infi }}$ :modules d'élasticité homogénéisés du bicouche « infi $»$.

$\mathrm{E}_{\mathrm{R}}^{\text {rayon }}, \mathrm{E}_{\mathrm{T}}^{\text {rayon }}, \mathrm{E}_{\mathrm{L}}^{\text {rayon }}$ : modules d'élasticité du tissu rayon ligneux.

$\mathrm{n}=\frac{\mathrm{v}_{\text {rayon }}}{\mathrm{V}_{\mathrm{T}}}$ fraction volumique en rayons ligneux, rapport $\mathrm{du}$ volume de rayons ligneux $\mathrm{V}_{\text {rayon }}$ au volume total $\mathrm{V}_{\mathrm{T}}$.

Les homogénéisations mises en œuvre dans les deux paragraphes précédents ne constituent certainement pas des solutions optimales à la recherche des propriétés élastiques des solides homogènes équivalents. Elles répondent toutefois à l'objectif de ce travail qui est moins de prévoir avec une précision sévère les propriétés du matériau bois à partir de ce celles des constituants, que de rendre compte avec pertinence de l'influence des différents paramètres pris en compte sur le comportement macroscopique observable, ceci à des fins de hiérarchisation de l'influence de ces paramètres.

\section{VALIDATION DES MODÈLES PRÉVISIONNELS DE L'ANISOTROPIE ÉLASTIQUE}

Valider la pertinence de la pyramide des modèles introduits au chapitre précédent pose deux classes de problèmes. Il convient, d'une part, de disposer de valeurs cibles cohérentes des propriétés macroscopiques élastiques que la modélisation est sensée simuler. Il faut, d'autre part, choisir un jeu réaliste de l'ensemble des paramètres utilisés par les différents modèles.

\subsection{Des propriétés matérielles cibles}

Les caractéristiques élastiques utilisées comme cibles pour valider la modélisation sont celles du « résineux standard ». Il s'agit des propriétés élastiques d'un bois résineux « virtuel », stabilisé à un taux d'humidité de $12 \%$ et de masse volumique $\rho=0,45 \mathrm{~g} \cdot \mathrm{cm}^{-3}$, statistiquement représentatif d'une banque de données expérimentales relatives à 38 essences résineuses [16].

Les caractéristiques retenues sont les modules d'élasticité suivant les trois directions d'orthotropie du bois, soit :

$\mathrm{E}_{\mathrm{R}}=1,00 \mathrm{GPa}$ Module d'élasticité radial ;

$\mathrm{E}_{\mathrm{T}}=0,636 \mathrm{GPa}$ Module d'élasticité tangentiel ;

$\mathrm{E}_{\mathrm{L}}=13,1 \mathrm{GPa}$ Module d'élasticité longitudinal.

Les rapports d'anisotropie $\mathrm{E}_{\mathrm{L}} / \mathrm{E}_{\mathrm{R}}=13,1$ et $\mathrm{E}_{\mathrm{R}} / \mathrm{E}_{\mathrm{T}}=1,57$, bien que redondants, sont de même considérés.

À des fins de comparaison avec les modules élastiques du résineux standard, les propriétés élastiques modélisées, correspondant à un bois vert, sont corrigées en humidité et ramenées du point de saturation des fibres, estimé ici à $\mathrm{H}_{\mathrm{PSF}}=30 \%$, à l'humidité de référence $\mathrm{H}=12 \%$, moyennant les relations données dans [16]:

$$
\mathrm{E}_{\mathrm{i}}^{\mathrm{H}}=\mathrm{E}_{\mathrm{i}}^{12}\left[1-\alpha_{\mathrm{i}}(\mathrm{H}-12)\right]
$$

avec respectivement $\alpha_{\mathrm{R}}=0,030(\% / \% \mathrm{H}), \alpha_{\mathrm{T}}=0,030(\% / \% \mathrm{H})$, $\alpha_{\mathrm{L}}=0,015(\% / \% \mathrm{H})$.

\subsection{Une base de donnée des paramètres structuraux}

Le tableau I regroupe pour des essences résineuses les valeurs de différents paramètres considérés, extraites des travaux d'une bonne vingtaine d' auteurs. Sur cette base un jeu probable de paramètres de départ a été défini. Les valeurs initiales choisies sont reportées dans l'avant dernière colonne du tableau I.

L'optimisation dont il est rendu compte ici a été conduite sous deux types de contraintes:

- Les angles des microfibrilles des tissus ligneux à l'intérieur du cerne ont été forcés de telles sorte qu'ils diffèrent et soient compris entre 5 et 10 degrés pour le bois final et supérieur à 20 degrés pour le bois initial, répondant ainsi à certaines propositions bibliographiques [1], [31].

- La masse volumique du bois résineux standard est fixée à $\rho_{\mathrm{b}}=0,45 \mathrm{~g} / \mathrm{cm}^{3}$.

Sachant que la masse volumique de chacun des tissus ( $\rho_{\text {initial }}$, $\left.\rho_{\text {final }}, \rho_{\text {rayon }}\right)$ est fixée par la donnée des paramètres géométriques de chacun de ceux-ci (4), la contrainte sur la masse volumique du bois est satisfaite en considérant que la fraction volumique en rayons ligneux $n$ et la texture $T_{X}$ sont tels que :

$$
\begin{gathered}
\rho_{b}=(1-n) \rho_{\text {infi }}+n \rho_{\text {rayon }} \\
\rho_{\text {infi }}=\left(1-T_{x}\right) \rho_{\text {initial }}+T_{x} \rho_{\text {final }} .
\end{gathered}
$$

Étant donné que $\rho_{b}$ est fixée, que n est donné et que $\rho_{\text {rayon }}$ est calculé, la relation (15) implique $P_{\text {infi }}$. De plus, $\rho_{\text {initial }}$ et $\rho_{\text {final }}$ ayant été calculés, alors la relation (16) implique la texture $\mathrm{T}_{\mathrm{x}}$. Tout ceci suppose que les données choisies soient réalistes pour conduire à des résultats cohérents.

\section{RÉSULTATS ET DISCUSSIONS}

\subsection{Procédure d'optimisation des paramètres structuraux}

L'ensemble des relations introduites dans les paragraphes précédents sont aisément saisies et traitées sur un tableur numérique de type Excel. Les différentes estimations des propriétés élastiques, arrondies à trois chiffres significatifs, ainsi que les valeurs des paramètres qui ont permis de les obtenir sont répertoriées dans les colonnes de $\mathrm{A}$ à $\mathrm{F}$ du tableau II.

L'analyse des résultats obtenus à chaque étape suggère des modifications des paramètres structuraux pour l'étape suivante. Cette procédure d'optimisation empirique, quelque peu archaïque, a été préférée à une méthode mathématique qui aurait conduit plus rapidement, mais en aveugle, au jeu de paramètres optimisés.

Les discussions et commentaires qui suivent, illustrent l'intérêt de la démarche, notamment sur le plan pédagogique. 
Tableau I. Base de données bibliographiques de paramètres structuraux.

\begin{tabular}{|c|c|c|c|c|c|c|c|c|c|c|c|c|c|c|c|c|c|c|c|c|c|c|c|}
\hline$\frac{\mathscr{E}}{\stackrel{\Xi}{z}}$ & 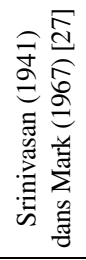 & 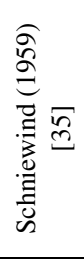 & 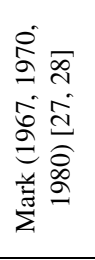 & 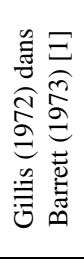 & 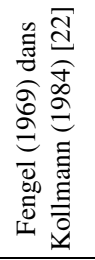 & 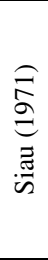 & 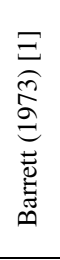 & 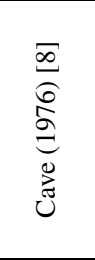 & 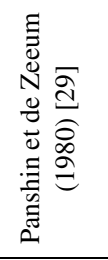 & 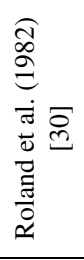 & 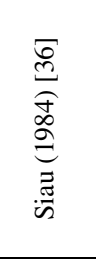 & 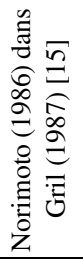 & 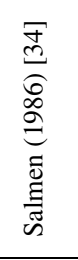 & 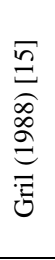 & 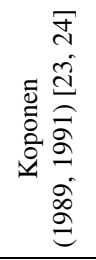 & 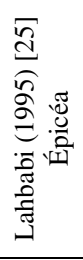 & 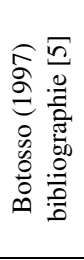 & 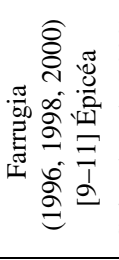 & 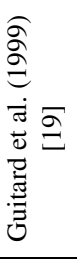 & 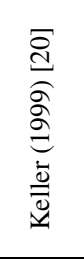 & 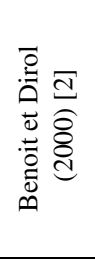 & 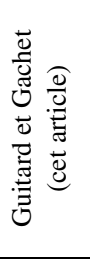 & 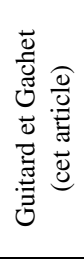 \\
\hline Em (Gpa) & 2 & - & $\begin{array}{c}1,23 \mathrm{et} \\
2,04\end{array}$ & - & - & - & 2 & - & - & - & - & 2 & - & 1,8 & - & - & - & & 1,2 & - & - & 2 & 2 \\
\hline $\mathrm{Nu}$ & - & - & 0,3 & 0,3 & - & - & 0,3 & - & - & - & - & - & 0,3 & 0,3 & - & - & - & - & 0,3 & - & - & 0,3 & 0,3 \\
\hline $\mathrm{V}(\%)$ & - & - & 50 & & - & - & - & - & - & - & - & 50 & - & 50 & - & - & - & - & 40 & - & - & 40 & 50 \\
\hline Ef (GPa) & - & - & - & - & - & - & 27 & - & - & - & - & - & - & - & - & - & - & - & 40 & - & - & 40 & 62 \\
\hline$\varphi_{\mathrm{i}}$ (degré) & - & - & - & & - & - & 40 & 15 à 30 & 10 à 30 & 10 & - & - & 20 & - & 10 à 40 & - & 20 & - & - & - & - & 25 & 22 \\
\hline $\mathrm{D}_{\mathrm{ti}}(\mu \mathrm{m})$ & - & - & - & & 25 à 38 & - & 36 & - & 30 à 45 & - & 15 à 80 & - & - & - & - & - & - & 25 & - & - & 20 à 60 & 30 & 30 \\
\hline $\mathrm{D}_{\mathrm{ri}}(\mu \mathrm{m})$ & - & - & - & & 30 à 50 & 35 & 34 & - & - & - & - & - & - & - & 30 & - & - & 34 & - & - & - & 35 & 37,5 \\
\hline $\mathrm{e}_{\mathrm{ti}}(\mu \mathrm{m})$ & - & - & - & & - & - & - & - & - & - & $2^{*}$ & - & $1-5^{*}$ & - & 1 à 2 & - & - & 3 & - & - & - & 2 & 1,068 \\
\hline $\mathrm{e}_{\mathrm{ri}}(\mu \mathrm{m})$ & - & - & - & & - & - & - & - & - & - & $2 *$ & - & $1-5^{*}$ & - & 1 à 2 & - & - & 4 & - & - & - & 3 & 0,854 \\
\hline$\varphi_{\mathrm{f}}($ degré $)$ & - & - & - & & - & - & 10 & 15 à 30 & 10 à 30 & 10 & - & - & 20 & - & 0 à 30 & - & 20 & - & - & - & - & 10 & 5,9 \\
\hline $\mathrm{D}_{\mathrm{tf}}(\mu \mathrm{m})$ & - & - & - & & 23 à 38 & - & 36 & - & 30 à 45 & - & 15 à 80 & - & - & - & 30 & - & - & 60 & - & - & 20 à 60 & 35 & 35 \\
\hline $\mathrm{D}_{\mathrm{rf}}(\mu \mathrm{m})$ & - & - & - & & 13 à 24 & 35 & 16 & - & - & - & - & - & - & - & 10 & - & - & 18 & - & - & - & 18 & 15 \\
\hline $\mathrm{e}_{\mathrm{tf}}(\mu \mathrm{m})$ & - & - & - & & - & - & - & - & - & - & $2^{*}$ & - & $1-5^{*}$ & - & 4 à 5 & - & - & 9 & - & - & - & 3 & 2,87 \\
\hline $\mathrm{e}_{\mathrm{rf}}(\mu \mathrm{m})$ & - & - & - & & - & - & - & - & - & - & $2^{*}$ & - & $1-5^{*}$ & - & 4 à 5 & - & - & 9 & - & - & - & 3 & 2,87 \\
\hline Vol.R.L (\%) & - & 17 & - & & - & - & 6 & - & 7 & - & 6 & - & - & - & 5 & - & - & 6 & - & 3 à 12 & - & 2 & 2 \\
\hline Texture (\%) & - & - & - & & - & - & - & - & - & - & - & - & - & & - & 20 & - & - & - & $\leq 50$ & $\leq 30$ & Optim & 51,2 \\
\hline
\end{tabular}

* Données globales ne faisant pas de distinction entre bois initial et bois final. 
Tableau II. Paramètres optimisés pour atteindre les caractéristiques élastiques cibles.

\begin{tabular}{|c|c|c|c|c|c|c|}
\hline Paroi de cellule bois initial & $\mathbf{A}$ & $\mathbf{B}$ & $\mathbf{C}$ & $\mathbf{D}$ & $\mathbf{E}$ & $\mathbf{F}$ \\
\hline $\mathrm{E}_{\mathrm{m}}$ matrice $\mathrm{GPa}$ & 1,200 & 1,200 & 1,200 & 1,200 & 2,000 & 0,550 \\
\hline$v$ coef. Poisson matrice & 0,300 & 0,300 & 0,300 & 0,300 & 0,300 & 0,350 \\
\hline $\mathrm{E}^{\mathrm{f}}$ fibrilles $\mathrm{GPa}$ & 40,000 & 64,000 & 64,000 & 64,000 & 62,000 & 61,500 \\
\hline AMF degrés & 25,000 & 25,000 & 25,000 & 25,000 & 22,000 & 12,300 \\
\hline V taux de microfibrilles & 0,500 & 0,500 & 0,500 & 0,500 & 0,500 & 0,500 \\
\hline \multicolumn{7}{|l|}{ Tissu de bois initial } \\
\hline $\mathrm{D}_{\mathrm{ti}}$ diamètre tangentiel en $\mu \mathrm{m}$ & 30,000 & 30,000 & 30,000 & 30,000 & 30,000 & 50,000 \\
\hline $\mathrm{e}_{\mathrm{ti}}$ épaisseur paroi tangentielle en $\mu \mathrm{m}$ & 2,000 & 2,000 & 1,000 & 1,000 & 1,068 & 2,350 \\
\hline OV paramètre d'ovalisation $\mathrm{D}_{\mathrm{ri}} / \mathrm{D}_{\mathrm{ti}}$ & 1,166 & 1,166 & 1,166 & 1,166 & 1,250 & 1,000 \\
\hline $\mathrm{e}_{\mathrm{i}}=\mathrm{e}_{\mathrm{ri}} / \mathrm{e}_{\mathrm{ti}}$ paramètre d'épaisseur pariétale & 1,500 & 1,500 & 1,500 & 1,500 & $\mathbf{0 , 8 0 0}$ & 1,000 \\
\hline$\rho_{\text {initial }}$ masse volumique en $\mathrm{g} / \mathrm{cm}^{3}$ & $\mathbf{0 , 4 4 0}$ & 0,440 & 0,229 & 0,229 & 0,167 & 0,271 \\
\hline \multicolumn{7}{|l|}{ Paroi de cellule bois final } \\
\hline $\mathrm{E}_{\mathrm{m}}$ matrice $\mathrm{GPa}$ & 1,200 & 1,200 & 1,200 & 1,200 & 2,000 & 0,550 \\
\hline$v$ coef. Poisson matrice & 0,300 & 0,300 & 0,300 & 0,300 & 0,300 & 0,350 \\
\hline$E^{f}$ fibrilles $\mathrm{GPa}$ & 40,000 & 64,000 & 64,000 & 64,000 & 62,000 & 61,500 \\
\hline AMF degrés & 10,000 & 10,000 & 10,000 & $\mathbf{8 , 5 0 0}$ & $\mathbf{5 , 9 0 0}$ & 12,300 \\
\hline V taux de microfibrilles & 0,500 & 0,500 & 0,500 & 0,500 & 0,500 & 0,541 \\
\hline \multicolumn{7}{|l|}{ Tissu de bois final } \\
\hline $\mathrm{D}_{\mathrm{tf}}$ diamètre tangentiel en $\mu \mathrm{m}$ & 35,000 & 35,000 & 35,000 & 35,000 & 35,000 & 50,000 \\
\hline $\mathrm{e}_{\mathrm{tf}}$ épaisseur paroi tangentielle en $\mu \mathrm{m}$ & 3,000 & 3,000 & 3,000 & 3,000 & 2,870 & 4,500 \\
\hline OV paramètre d'ovalisation $\mathrm{D}_{\mathrm{rf}} / \mathrm{D}_{\mathrm{tf}}$ & 0,514 & 0,514 & 0,514 & 0,514 & $\mathbf{0 , 4 3 0}$ & $\mathbf{0 , 4 4 0}$ \\
\hline $\mathrm{e}_{\mathrm{f}}=\mathrm{e}_{\mathrm{rf}} / \mathrm{e}_{\mathrm{tf}}$ paramètre d'épaisseur pariétale & 1,000 & 1,000 & 1,000 & 1,000 & 1,000 & 1,000 \\
\hline$\rho_{\text {final }}$ masse volumique en $\mathrm{g} / \mathrm{cm}^{3}$ & 0,676 & 0,676 & 0,676 & 0,676 & 0,729 & 0,778 \\
\hline \multicolumn{7}{|l|}{ Paroi de cellule de rayon } \\
\hline $\mathrm{E}_{\mathrm{m}}$ matrice $\mathrm{GPa}$ & 1,200 & 1,200 & 1,200 & 1,200 & 2,000 & 0,550 \\
\hline$v$ coef. Poisson matrice & 0,300 & 0,300 & 0,300 & 0,300 & 0,300 & 0,350 \\
\hline $\mathrm{E}^{\mathrm{f}}$ fibrilles $\mathrm{GPa}$ & 40,000 & 64,000 & 64,000 & 64,000 & 60,000 & 61,500 \\
\hline AMF degrés & 40,000 & 40,000 & 40,000 & 40,000 & 46,000 & 12,300 \\
\hline V taux de microfibrilles & 0,500 & 0,500 & 0,500 & 0,500 & 0,500 & 0,500 \\
\hline \multicolumn{7}{|l|}{ Tissu de rayon } \\
\hline $\mathrm{D}_{\mathrm{tr}}$ diamètre tangentiel en $\mu \mathrm{m}$ & 30,000 & 30,000 & 30,000 & 30,000 & 34,500 & 50,000 \\
\hline $\mathrm{e}_{\mathrm{tr}}$ épaisseur paroi tangentielle en $\mu \mathrm{m}$ & 2,000 & 2,000 & 2,000 & 2,000 & 1,032 & 2,400 \\
\hline OV paramètre d'ovalisation $D_{\mathrm{rr}} / \mathrm{D}_{\mathrm{tr}}$ & 1,166 & 1,166 & 1,166 & 1,166 & 1,180 & 1,000 \\
\hline$e_{R}=e_{r r} / e_{t r}$ paramètre d'épaisseur pariétale & 1,500 & 1,500 & 1,500 & 1,500 & 1,500 & 1,000 \\
\hline$\rho_{\text {rayon }}$ masse volumique en $\mathrm{g} / \mathrm{cm}^{3}$ & 0,440 & 0,440 & 0,440 & 0,440 & 0,202 & 0,276 \\
\hline \multicolumn{7}{|l|}{ Paramètres de cerne } \\
\hline $\mathrm{T}_{\mathrm{x}}$ texture & 0,043 & 0,043 & 0,495 & 0,495 & 0,512 & 0,360 \\
\hline $\mathrm{n}$ fraction volumique en rayons en $\%$ & 2,000 & 2,000 & 2,000 & 2,000 & 2,000 & 1,750 \\
\hline \multicolumn{7}{|l|}{ Les critères cibles } \\
\hline Masse volumique $=0,45$ & 0,45 & 0,45 & 0,45 & 0,45 & 0,45 & 0,450 \\
\hline$E_{R}=1,00 ~ G P a$ & 2,950 & 4,46 & 1,75 & 1,55 & 1,00 & 0,995 \\
\hline $\mathrm{E}_{\mathrm{T}}=\mathbf{0 , 6 3 6} \mathbf{G P a}$ & 0,360 & 0,545 & 0,758 & 0,621 & 0,635 & 0,634 \\
\hline $\mathrm{E}_{\mathrm{L}}=\mathbf{3 , 1} \mathbf{G P a}$ & 8,16 & 12,9 & 13,1 & 13,1 & 13,1 & 13,2 \\
\hline $\mathrm{E}_{\mathrm{L}} / \mathrm{E}_{\mathrm{R}}=\mathbf{1 3 , 1}$ & 2,76 & 2,89 & 7,50 & 8,47 & 13,1 & 13,3 \\
\hline $\mathrm{E}_{\mathrm{R}} / \mathrm{E}_{\mathrm{T}}=\mathbf{1 , 5 7}$ & 8,20 & 8,17 & 2,31 & 2,50 & 1,57 & $\mathbf{1 , 5 7}$ \\
\hline
\end{tabular}




\subsection{Vers un jeu de paramètres caractéristiques du résineux standard}

La colonne A du tableau II correspond au jeu initial des paramètres structuraux. Ces premiers résultats suggèrent trois remarques essentielles:

- le module d'élasticité $\mathrm{E}_{\mathrm{L}}=8,16 \mathrm{GPa}$ est $40 \%$ plus faible que la valeur cible ; [20],

- la texture $\mathrm{T}_{\mathrm{x}}$ de l'ordre de $4 \%$ est anormalement basse

- les rapports d'anisotropie obtenus ne sont pas réalistes.

Un premier ajustement a été d'augmenter la rigidité $\mathrm{E}^{\mathrm{f}} \mathrm{des}$ microfibrilles, en proportion du déficit constaté sur la valeur de $\mathrm{E}_{\mathrm{L}}$. L'efficacité de ceci est traduit dans la colonne $\mathrm{B}$ où $\mathrm{E}_{\mathrm{L}}=$ $12,9 \mathrm{GPa}$.

Une masse volumique de bois initial de $\rho_{\text {initial }}=0,44 \mathrm{~g} / \mathrm{cm}^{3}$ est trop élevée. En référence au profil microdensitométrique donné en figure 6 qui indique une valeur inférieure à $0,3 \mathrm{~g} / \mathrm{cm}^{3}$, la seconde modification a porté sur une diminution de l'épaisseur pariétale du bois initial, à diamètre cellulaire inchangé. L'incidence de cette seconde modification est illustrée en colonne $\mathrm{C}$. Il en résulte une texture $\mathrm{T}_{\mathrm{X}}=48 \%$ plus réaliste et la relation d'ordre entre les modules d'élasticité est maintenant respectée $\left(\mathrm{E}_{\mathrm{T}}<\mathrm{E}_{\mathrm{R}}<<\mathrm{E}_{\mathrm{L}}\right)$. Si l'estimation de $\mathrm{E}_{\mathrm{L}}$ est satisfaisante, celle de $\mathrm{E}_{\mathrm{T}}$ et surtout de $\mathrm{E}_{\mathrm{R}}$ sont encore très supérieures aux valeurs cibles, respectivement, de 20 et de $75 \%$.

À travers les résultats de la colonne D, c'est la sensibilité de la modélisation à l'angle des microfibrilles qui est illustrée. La troisième modification envisagée porte sur l'AMF de la seule paroi de bois final, qui passe de $\varphi=10$ à 8,5 degrés. La sensibilité des caractéristiques élastiques à ce paramètre est très forte. Une réduction de 1,5 degré de l'AMF, (depuis 10 degrés), induit une diminution de $11 \%$ de $\mathrm{E}_{\mathrm{R}}$ et de $18 \%$ de $\mathrm{E}_{\mathrm{T}}$. Le module $\mathrm{E}_{\mathrm{L}}$ est en revanche pratiquement insensible à cette modification.

\subsection{Commentaires sur les paramètres optimisés}

La colonne E du tableau II rend compte d'un jeu de paramètres structuraux optimisés, obtenus moyennant le critère d'un écart relatif de chaque des constante élastique calculée, à la valeur cible correspondante, inférieur de $1 \%$. Les contraintes étant celles indiquées au paragraphe 3.2, à savoir une masse volumique du bois fixée à $\rho_{b}=0,45 \mathrm{~g} \cdot \mathrm{cm}^{-3}$ et un AMF significativement différent dans le bois initial et dans le bois final.

Une première remarque porte sur le module d'élasticité des microfibrilles, dont la valeur optimisée $\mathrm{E}^{\mathrm{f}}=62 \mathrm{GPa}$ est sensiblement la moitié du module d'élasticité de $134 \mathrm{GPa}$ affiché par Sakurada et al. [32]. Deux commentaires explicatifs permettent de justifier cette différence.

En premier lieu, la modélisation micromécanique du renforcement de la paroi cellulaire par des microfibrilles de cellulose que nous utilisons ici [19], défini par les relations (1) et (2), diffère des modèles classiquement mis en œuvre pour évaluer les propriétés des fibres, par méthode inverse, à partir de mesures de rigidité de tissus ligneux. Notre modèle assure la « restriction » du cisaillement induit entre deux cellules voisines vraies en envisageant une cellule élémentaire fictive constituée d'un squelette tridimensionnel de microfibrilles noyé dans une matrice

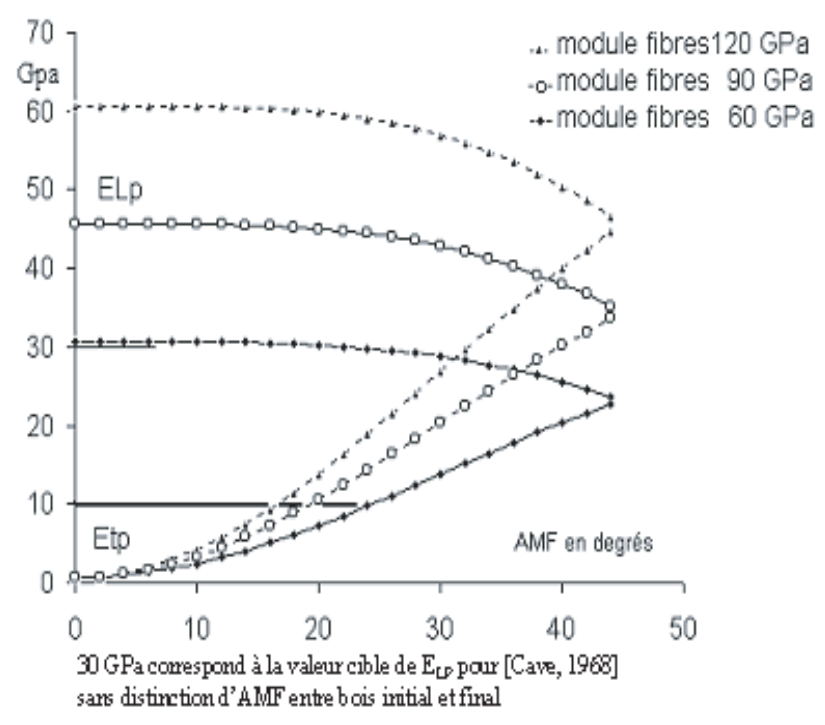

$10 \mathrm{GPa}$ estla valeur able de $\mathrm{E}_{\pi \mathrm{C}}$ [C are, 1998], [Khale et al, 1994] sars distinction d'AMF entre bois iritial et final

Pour du bois iritial de Piceo Akies, [Bergander et al, 2000] affichenture valeur expérimentale de $2,5 \mathrm{GPa}$ pour $\mathrm{E}_{\mathrm{TP}}$

Figure 8. Évolution des modules élastiques pariétaux $\mathrm{E}_{\mathrm{LP}}$ et $\mathrm{E}_{\mathrm{TP}}$ en fonction de l'angle des microfibrilles.

amorphe. Ce modèle est rigidifiant en regard de celui d'une structure multicouche renforcée par des nappes de fibres parallèles disjointes. La figure 8 illustre la sensibilité des modules élastiques pariétaux longitudinal $\mathrm{E}_{\mathrm{LP}}$ et transverse $\mathrm{E}_{\mathrm{TP}}$ à l' angle de microfibrilles. On constate notamment la rigidification du module d'élasticité transverse avec une augmentation de l'AMF.

En second lieu, un module de $130 \mathrm{GPa}$ est une évaluation des propriétés élastiques de la cristallite de cellulose obtenue par des mesures de déplacement de plans réticulaires par diffraction rayons $\mathrm{X}$. Il est admis que les éléments constitutifs des microfibrilles sont des filaments comportant des zones de cellulose fortement cristallisé (cristallite) intimement associées avec des zones de cellulose amorphe [12, 27] voire des incrustations lenticulaire de lignine mêlée d'hémicelluloses.

Ces deux arguments convergent ici pour expliquer que le module élastique apparent optimisé des microfibrilles soit plus faible que le module de fibrilles purement cristallines.

Le bois « virtuel » correspondant à ce jeu de paramètres est fortement hétérogène quand à la masse volumique des tissus constitutifs, avec $0,73 \mathrm{~g} \cdot \mathrm{cm}^{-3}$ pour le bois final et $0,167 \mathrm{~g} \cdot \mathrm{cm}^{-3}$ pour le bois initial, en accord avec le profil microdensitométrique de la figure 6 .

Une seconde hétérogénéité tient à la différence d'angle des microfibrilles, avec une valeur d'AMF de 22 degrés pour le bois initial contre 5,9 degrés pour le bois final. Notons que ceci répond à l'une des contraintes imposées lors du processus d'optimisation.

La différence d'ovalisation des cellules entre bois initial et bois final est un troisième facteur hétérogénéité. Le rapport $\mathrm{D}_{\mathrm{R}} / \mathrm{D}_{\mathrm{T}}$ 
évolue en effet de 1,25 pour le bois initial à 0,43 pour le bois final. Ce résultat est sensiblement conforme à ce que suggère la micrographie figure 2a et la littérature [26].

Les paramètres de cerne, une texture de l'ordre de $50 \%$ et une fraction volumique en rayons ligneux de $2 \%$, fixent les proportions des tissus ligneux constitutifs du cerne. Avec $2 \%$, la proportion en rayons ligneux est probablement une valeur un peu faible.

En conclusion, le jeu de paramètres optimisés de la colonne E constitue un ensemble cohérent susceptible de caractériser un bois résineux virtuel de référence.

\subsection{Multiplicité des solutions}

Un second jeu de paramètres optimisés, présenté dans la colonne F, a été obtenu en levant la contrainte précédante sur l'AMF, l'orientation des microfibrilles est dans ce cas identique dans les trois tissus constitutifs de l'ordre de 12,3 degrés.

L'expérience numérique confirme la possibilité d'une multiplicité de solutions optimales. Il est en conséquence possible de fixer un certain nombre de contraintes d'optimisation devant conduire aux différents jeux de paramètres associés à chacun des types de bois observables au sein d'un même arbre (bois juvénile, bois adulte, bois de réaction).

\section{CONCLUSIONS ET PERSPECTIVES}

L'anisotropie élastique du bois « résineux standard » est simulée par empilement de trois échelles de modélisations :

- Cinq (5) paramètres sont utilisés pour décrire l'anisotropie élastique de la paroi cellulaire, dont : deux modules $\left(\mathrm{E}^{\mathrm{m}}, \mathrm{v}\right)$ caractérisent la matrice élastique homogène isotrope, deux paramètres qualifient l'élasticité $\left(E^{f}\right)$ et l'orientation $(\varphi)$ des microfibrilles de cellulose, le dernier paramètre $(\mathrm{V})$ fixe la proportion fibre-matrice.

- Quatre paramètres $\left(D_{t}, e_{t}, D_{r}, e_{r}\right)$ décrivent la morphologie cellulaire d'un tissu ligneux, assimilé à une structure nid d'abeille constituée de cellules rectangulaires dont les parois radiales sont supposées alignées, tandis que les parois tangentielles sont disposées en quinconce ; ils caractérisent le diamètre cellulaire et l'épaisseur de paroi dans les directions radiale et tangentielle.

- Trois tissus ligneux constituent le cerne de croissance dont la composition est réglée par deux paramètres, la texture $\left(T_{X}\right)$ qui fixe la proportion de bois initial et de bois final et la fraction volumique (n) en rayon ligneux.

C'est, en conséquence, un modèle à 17 paramètres qui est mis en œuvre pour simuler l'anisotropie élastique du bois, caractérisée ici par les modules d'élasticité dans chacune des directions d'orthotropie du matériau $\mathrm{E}_{\mathrm{R}}, \mathrm{E}_{\mathrm{T}}, \mathrm{E}_{\mathrm{L}}$.

Un jeu cohérent de paramètres optimisés a été obtenu pour décrire le « résineux standard » sous deux types de contraintes ; une masse volumique du bois fixée à $\rho_{b}=0,45 \mathrm{~g} \cdot \mathrm{cm}^{-3}$ et des angles de microfibrilles différenciées entre bois initial (< 10 degrés) et bois final (> 20 degrés).

Ce modèle constitue ainsi un apport méthodologique dans la description de l'anisotropie du bois. Il se distingue notamment des approches multi échelles classiques (caractérisant le passage micro macro) par sa démarche de type « gigogne » comme par l'adoption d'un jeu de paramètres descriptifs strictement utiles. Ces simplifications permettant de rendre compte de l'anisotropie transverse, les auteurs se sont attachés à conserver une cohérence interdisciplinaire dans les niveaux de finesses de description. Le caractère évolutif de ces niveaux permettra l'intégration éventuelle de facteurs complémentaires améliorant d'autant en terme biologique le réalisme de la modélisation.

L'emploi, à l'échelle ultrastructurale, du modèle « squelette de microfibrilles », plus rigidifiant en regard des approches pariétales multicouches, a permis de considérer la sous couche $\mathrm{S}_{2}$, caractérisée ici par le paramètre angle des microfibrilles, comme majoritairement responsable de l'anisotropie pariétale, et a rendu ainsi l'intervention des autres sous couches $S_{1}$ et $S_{2}$ non strictement nécessaire au sens mécanique.

Cet outil de simulation ouvre des possibilités d'expérimentation numérique en appui aux campagnes expérimentales traditionnelles de caractérisation du matériau bois, notamment en permettant :

- une analyse détaillée de la sensibilité des propriétés élastiques macroscopiques à la variabilité des différents paramètres structuraux et ultrastructuraux,

- d'associer à la diversité des bois, présent au sein d'une même grume, des jeux de paramètres microstructuraux spécifiques, en tenant compte de contraintes d'optimisations découlant d'observations expérimentales,

- d'inspirer, à travers la méthodologie mise en œuvre, des extensions de la modélisation vers des domaines connexes, notamment, touchant au comportement thermo-hygro-viscoélastique, aux variations dimensionnelles hygro-thermiques, à la caractérisation de la perméabilité et de diffusivité en phase liquide et gazeuse,

- d'étendre la démarche vers un champ d'investigation beaucoup plus complexe, celui des essences feuillues. Dans cette perspective, la complexité structurale évidente des essences feuillues impliquera de substituer à certains changements d'échelle envisagés ici des modèles parfois moins simplistes [37].

\section{RÉFÉRENCES}

[1] Barrett J.D., Theorical models of wood shrinkage and elasticity. $\mathrm{Ph} . D$. University of California, Berkeley, 1973.

[2] Benoit Y., Dirol D., Le guide de reconnaissance des bois de France, CTBA, Éditions Eyrolles, 1999.

[3] Bergander A., Salmen L., The transverse elastic modulus of the native wood fibre wall, J. Pulp Pap. Sci. 26 (2000) 234-238.

[4] Bodig J., Jayne B.A., Mechanics of wood and wood composites, Van Nostrand Reinhold, New York, NY, 1982.

[5] Botosso P.C., Une méthode de mesure du retrait microscopique du bois : Application à la prédiction du retrait tangentiel d'éprouvettes de bois massif de Sapin pectiné (Abies alba Mill.), thèse Nancy I, 1997.

[6] Cave I.D., The anisotropic elasticity of the plant cell wall, Wood Sci. Technol. 2 (1968) 268-278.

[7] Cave I.D., Wood substance as a water reactive fibre reinforced composite, J. Microsc. 104 (1975) 47-52.

[8] Cave I.D., Modelling the structure of softwood cell wall for computation of mechanical properties, Wood Sci. Technol. 10 (1976) 19-28. 
[9] Farrugia F., Détermination du comportement élastique d'un ensemble de fibres de bois à partir de son organisation cellulaire et d'essais mécaniques sous microscope, thèse ENGREF, Nancy, 1998.

[10] Farrugia F., Lahbabi R., Perré P., Détermination des propriétés mécaniques des résineux à l'échelle microscopique du plan ligneux, Cahiers Lorrains du Bois ARBOLOR, 1996.

[11] Farrugia F., Perre P., Microscopic tensile tests in the transverse plane of earlywood and latewood parts of spruce, Wood Sci. Technol. 34 (2000) 65-82.

[12] Fengel D., Wegener G., The internal structure of fibrils, in: Wood: Chemistry, Ultrastructure, Reactions, De Gruyter, Berlin, New York, 1984, pp. 97-98.

[13] Gibson L.J., Ashby M.F., Cellular solids, Pergamon Press, 1988.

[14] Gillis P.P., Orthotropic elastic constant of wood, Wood Sci. Technol. 6 (1972) 138-156.

[15] Gril J., Une modèlisation du comportement hygro-rhéologique du bois à partir de sa microstructure, thèse Université Paris VI, Paris, 1988.

[16] Guitard D., Mécanique du matériau bois et composites, Éditions Cepadues, 1987.

[17] Guitard D., El Amri F., Modèles prévisionnels du comportement élastique tridimensionnel des bois feuillus ou résineux, Ann. Sci. For. 44 (1987) 335-358.

[18] Guitard D., El Amri F., La fraction volumique en rayons ligneux comme paramètre explicatif de la variabilité de l'anisotropie élastique du matériau bois. Actes du $2^{\mathrm{e}}$ Colloque des Sciences et Industries du Bois, 22-24 Avril 1987, Nancy.

[19] Guitard D., Masse H., Yamamoto H., Okuyama T., Growth stress generation: a new mechanical model of the dimensional change of wood cells during maturation, J. Wood Sci. 45 (1999) 384-391.

[20] Keller R., Cours de DEA Sciences du Bois: structure, composition, formation du bois, ENGREF, 1999.

[21] Khale E., Woodhouse J., The influence of cell geometry on the elasticity of softwood, J. Mater. Sci. 29 (1994) 1250-1259.

[22] Kollmann F.P., Cote W.A., Principles of wood sciences and technology. 1. Solid wood, Springer-Verlag, New York, 1984.

[23] Koponen S., Toratti T., Kanerva P., Modelling elastic and shrinkage properties of wood, Wood Sci. Technol. 23 (1989) 55-63.
[24] Koponen S., Toratti T., Kanerva P., Modelling longitudinal elastic and shrinkage of wood based on cell structure, Wood Sci. Technol. 25 (1991) 25-32.

[25] Lahbabi R., Mesure par analyse d'image de paramètres microscopiques requis pour une prédiction déterministe des propriétés du bois, Thèse de doctorat de l'ENGREF, Nancy, 1995.

[26] Marion C., Caractérisation de l'agencement cellulaire du bois à l'aide de critères morphologiques mesurables par analyse d'images, Rapport de DEA Sciences du Bois, 2001.

[27] Mark R.E., Cell wall mechanics of tracheids, New Haven and London, Yale University Press, 1967.

[28] Mark R.E., Molecular and cell wall structure of wood. Adhesion in Cellulosic and Wood-Based Composites, Plenum Press, 1980, pp. 7-51.

[29] Panshin A.J., De Zeeuw C., Textbook of wood technology, 4th ed., Mc Graw Hill Book Company, 1980.

[30] Roland J.C., Mosiniak M., Czaninsky Y., Vian B., Précisions ultrastructurales sur les modalités d'oscillation dans l'orientation de la cellulose des parois du bois, Colloque Sciences et Industries du Bois, Groupe 1, Grenoble, 20-22 septembre 1982.

[31] Sahlberg U., Salmen L., Oscarsson A., The fibrillar orientation in the $S_{2}$ layer of wood fibres as determined by X-ray diffraction analysis, Wood Sci. Technol. 31 (1997) 77-86.

[32] Sakurada I., Nukushina Y., Ito T., Experimental determination of the elastic modulus of crystalline regions in oriented polymers, J. Polym. Sci. 57 (1962) 651-660.

[33] Salmen L., The cell wall as a composite structure, in: Bristow J.A (Ed.), Paper, Structure and properties, Editions Marcel Dekker, inc., New York, Basel, International Fiber Science and Technology Series 8, 1986, pp. 51-73.

[34] Salmen L., Kolseth P., A mechanical model of softwood, in: Bristow J.A. (Ed.), Paper, Structure and properties, Editions Marcel Dekker, inc., New York, Basel, International Fiber Science and Technology Series 8, 1986, pp. 377-380.

[35] Schniewind A.P., Transverse anisotropy of wood: A function of gross anatomic structure, For. Prod. J. (1959) 350-359.

[36] Siau J.F., Transport processes in wood, Springer-Verlag, Berlin N.Y., 1984.

[37] Yamamoto H., Sassus F., Ninomoiya M., Gril J., A model of anisotropic swelling and shrinking process of wood, Wood Sci. Technol. 35 (2001) 167-181. 